\title{
La conciliación como proceso transformador de relaciones en conflicto
}

\author{
Miguel Ángel Montoya Sánchez** \\ Natalia Andrea Salinas Arango***
}

Recibido: julio de 2016

Aprobado: octubre de 2016

DOI: 10.22395/ojum.v15n30a6

\section{RESUMEN}

En este escrito, resultado de la referida investigación, se presenta, desde una perspectiva diferente, el tratamiento de la conciliación como un proceso autocompositivo de resolución de conflictos, soportado su espíritu en el marco de sus fundamentos teóricos y el poder transformador de dicho mecanismo y no meramente desde el valor de simple instrumento de descongestión de despachos judiciales de que fuera dotado por el legislador. De este potencial, según los hallazgos de la investigación, también se dará cuenta desde las experiencias de los usuarios y conciliadores del Centro de Conciliación objeto de la misma.

Palabras clave: conciliación; mecanismos de resolución de conflictos; enfoque transformativo; interaccionismo simbólico.

\footnotetext{
Artículo resultado de la investigación terminada "La conciliación en el proceso de transformación del conflicto: a propósito de la conciliación en el Centro de Conciliación Luis Fernando Vélez Vélez de la Universidad de Antioquia", inscrita ante el Centro para el Desarrollo de la Investigación de la Universidad de Antioquia -CODI-; publicada en Montoya, Salinas, Osorio \& Martínez (2011).

* Abogado, Conciliador, especialista en Derecho de Familia, magíster en Derecho de la Universidad de Antioquia profesor titular de la Facultad de Derecho y Ciencias Políticas de la Universidad de Antioquia. Coordinador del Área de Derecho de Familia y del Semillero de Investigación en Derecho de Familia de dicha Facultad. Miembro del Grupo de Investigación "Derecho y Sociedad", Línea Tratamiento de conflictos. E-mail: miguel. montoya@udea.edu.co.

.*. Trabajadora social de la Universidad de Antioquia, magíster en Historia de la Universidad Nacional de Colombia, profesora asociada de la Universidad Pontificia Bolivariana. Coordinadora del Grupo de investigación de Trabajo Social -GITS-y Coordinadora del Semillero Dinámica Social de la UPB. E-mail: natalia.salinas@ upb.edu.co
} 


\title{
Conciliation as a Transformation Process of Conflictive Relations
}

\begin{abstract}
This article, result of a research, is presented from a perspective different from the treatment of conciliation as process comprising resolution of conflicts; its spirit is supported by its theoretical fundamentals and the transformation power of such mechanisms and not only as an ordinary instrument to relieve overcrowding of legal offices. From this potential, and according to the findings of the research, the article will also account for the experiences of users and conciliators from the Conciliation Center subject matter of such research.
\end{abstract}

Key words: conciliation; conflict resolution mechanisms; transformational approach; symbolic interaction. 


\section{INTRODUCCIÓN}

A raíz del trabajo desarrollado durante los últimos años con los usuarios del Consultorio Jurídico de la Facultad de Derecho y Ciencias Políticas de la Universidad de Antioquia, se han venido visibilizando fenómenos y hechos, que, aunque se podrían asumir como obvios, aportan algunas claridades importantes, que desde la academia se pueden entregar respecto del, a veces, mal entendido binomio teoría-práctica. Con el presente artículo se pretende, pues, dar cuenta de algunos resultados de la investigación que hace referencia al fenómeno de la conciliación, los fundamentos teóricos que la soportan y su praxis.

Se pretende, entonces, dar razón del potencial que guarda la conciliación como proceso autocompositivo de resolución de conflictos, soportado su espíritu en el marco de sus fundamentos teóricos y en su poder transformador y no meramente desde su valor de simple mecanismo de descongestión de despachos judiciales. Así, de dicho potencial también se dará cuenta desde las experiencias recuperadas en los usuarios y conciliadores en el Centro de Conciliación "Luis Fernando Vélez Vélez", de la Universidad de Antioquia.

La metodología utilizada en la investigación aludida se apoyó en la modalidad de estudio de caso, la cual permitió conocer las singularidades de las situaciones que presentan las personas y la forma en que se aborda la resolución de su conflicto, mediante la conciliación en dicho Centro de Conciliación. El interaccionismo simbólico (Schaefer, 2006; Ritzer, 2002) como enfoque metodológico, permitió la lectura y análisis de las construcciones simbólicas que establecen los actores sociales entre sí y las situaciones cotidianas que inciden en su comportamiento, actitudes e interacciones, en este caso, en el escenario del proceso conciliatorio (García, 2002).

Igualmente, se llevaron a cabo varias técnicas de investigación, como entrevistas semiestructuradas, un grupo focal y observaciones participantes no activas, las cuales aportaron información para su posterior análisis cualitativo, mediante la triangulación de las fuentes y posterior validación de los hallazgos presentados en el estudio.

A efectos de lo presentado en este artículo, se retoman algunos de los objetivos planteados en la investigación: describir los conflictos que se consultan y la forma de resolverlos en el Centro de Conciliación "Luis Fernando Vélez Vélez" de la Universidad de Antioquia, como objetivo principal; y describir el proceso que se asume para llevar a cabo las audiencias de conciliación y la forma como las personas asumen el conflicto y su resolución en este Centro, entre los objetivos específicos.

En primer lugar, se presentan algunos fundamentos de la conciliación, en la idea de enmarcarlos más allá del entendimiento de este como una mera técnica o mecanismo de descongestión y en los fundamentos de una teoría de la conciliación, tomados de los elementos 
apuntalados por Bush y Folger (1994) en el tratamiento específico de la mediación desde el denominado enfoque transformativo contrastado, al final, con el trabajo empírico realizado en la investigación con los conciliadores y los elementos aportados por los usuarios del Centro de Conciliación en su calidad de partes en los procesos de conciliación analizados.

En segundo lugar, se presenta la conciliación como un proceso mediante el cual se abren las posibilidades de transformación de los conflictos y de las relaciones de los sujetos vinculados con el conflicto mismo.

Y por último, se dará cuenta del proceso que se asume para llevar a cabo las audiencias de conciliación y de lo identificado sobre la noción que los usuarios y conciliadores tenían sobre los conceptos "conflicto", "conciliación", "comunicación o diálogo", "resolución" y/o "transformación" en el tratamiento de los conflictos, los cuales se fueron transformando durante el proceso pedagógico y en las audiencias conciliatorias, pues, y aun en el evento de que no se llegara necesariamente a un acuerdo, en particular, los usuarios terminaron sintiéndose más cercanos a la significación e importancia del encuentro con el otro, en la idea de la construcción de una determinada convivencia pacífica en sus relaciones cotidianas.

\section{LA CONCILIACIÓN, MÁS ALLÁ DE TÉCNICA O MECANISMO DE DESCONGESTIÓN JUDICIAL}

En Colombia, desde la exposición de motivos de la Ley 23 de 1991, se plan- teaba por parte de los proponentes de la iniciativa que la idea fundamental que inspiraba al proyecto era la de que los jueces solo debían ocuparse de los litigios para los cuales la sociedad no encontraba solución adecuada (Oñate, 1994, citado por Montoya, Salinas, Osorio \& Martínez, 2011). Así, y a sabiendas de que el fundamento de la expedición de dicha ley fue la descongestión de los despachos judiciales, los avances alcanzados desde la doctrina y la jurisprudencia respecto de la conciliación, cada vez más, han evidenciado, desde su práctica o aplicación técnica, la importancia de dicha figura más allá de la descongestión.

Sumando elementos a dicha argumentación, en la exposición de motivos de la Ley 446 de 1998 (República de Colombia, 1997), se resalta la importancia de la resolución de los conflictos emanada directamente de los implicados, cuando se dice que,

[...] resulta posible pensar que muchas personas, para quienes la Justicia tradicional no actúa de manera ágil y efectiva o no responde eficazmente a su necesidad de solucionar problemas de carácter jurídico, acudan a soluciones ajenas a la institucionalidad y, en muchas ocasiones, generadoras de mayores conflictos. Por lo anterior se evidencia la necesidad, cada vez mayor, de dotar al Estado y a los particulares de medios que les permitan encarar sus conflictos en forma más positiva, ya que desde antiguo se ha reafirmado que la ley del talión no debe encontrar eco dentro de las sociedades civilizadas (p. 28). 
Y agrega,

[...] así, se evidencian las numerosas bondades que han venido ofreciendo los sistemas alternos de solución de conflictos, los cuales -por ese motivo- se estiman de gran importancia en el presente proyecto, pues constituyen útiles herramientas para un segmento significativo de la población que percibe como distantes los mecanismos formales de la Justicia, representando así una salida jurídica a la falta de acceso al derecho y la Justicia que evite la exclusión y marginalidad social en esta materia (p. 37).

En este sentido, la comprensión del concepto de Teoría de la conciliación que se pretende evidenciar en el presente escrito no ha sido ajeno a los múltiples pronunciamientos que sobre ello ha realizado la Corte Constitucional. En la Sentencia C-1195 de 2001 (Colombia, Corte Constitucional, 2001), se dice, por ejemplo:

Para la Corte resulta claro que la justicia estatal formal no siempre es efectiva, en especial cuando no se han previsto recursos judiciales idóneos y suficientes que faciliten la solución pacífica de los conflictos, o cuando la complejidad de los procedimientos o de las condiciones de tiempo, modo y lugar exigidas por el legislador restringen la capacidad de alcanzar el goce efectivo de los derechos cuya protección se busca al acudir a las instancias judiciales. Los mecanismos alternativos de resolución de conflictos no representan una desconfianza hacia la justicia estatal formal, sino un reconocimiento de que procedimientos menos formales y alternativas de justicia autocompositiva complementan las opciones a las cuales pueden acudir las personas para resolver sus disputas. Por ello, mecanismos como la mediación y la conciliación, más que medios para la descongestión judicial, son instrumentos para garantizar el acceso efectivo a la justicia y promover la resolución pacífica de los conflictos (p. 29).

Y termina diciendo,

La conciliación contribuye a la consecución de la convivencia pacífica, uno de los fines esenciales del Estado. El hecho de que a través de la conciliación sean las partes, con el apoyo de un conciliador, las que busquen fórmulas de acuerdo para la solución de un conflicto, constituye una clara revelación de su virtud moderadora de las relaciones sociales. La conciliación extrae, así sea transitoriamente, del ámbito litigioso la resolución de los conflictos, allanando un camino para que las disputas entre individuos se resuelvan por la vía del acuerdo. Además, la conciliación estimula el diálogo, reduce la cultura adversarial y elimina la agudización del conflicto como consecuencia del litigio (p. 40).

Lo que se aprecia en el espíritu de la norma es que la conciliación trasciende la preocupación de descongestionar los despachos judiciales, puesto que lo que en verdad se evidencia es la capacidad que tienen las personas vinculadas con el 
conflicto de juntarse y cooperativamente resolver los conflictos, en el escenario de interacción que le caracteriza y que propicia el establecimiento de ciertas formas propositivas de concebir el conflicto y su consecuente transformación.

A partir de lo anterior, se hace necesario extender las bases que soportan este recurso jurídico de tratamiento amigable de los conflictos, a sus principios basilares o fundamentación teórica. Así pues, la epistemología de la conciliación, se podría decir, no es otra que la epistemología del conflicto, máxime que en el plano de lo jurídico no subsistiría la primera sin el segundo, pues allí, la percepción del mundo por quienes hacen parte de una cierta comunidad no deja de ser, por alguna circunstancia conflictiva que los relacione, la del choque de intereses o de derechos que hay que intentar resolver, siempre, en presencia de un tercero que hará las veces de facilitador.

La teoría de la conciliación bien podría entenderse entonces, como el proceso de comunicación interaccional, amplio, simétrico y directo que se establece entre personas vinculadas por un conflicto y mediadas por un tercero, a través del cual se suscita el encuentro a efectos de su posible resolución o transformación.

\section{LA CONCILIACIÓN. PROCESO DE TRANSFORMACIÓN}

Desde la etimología, el término conciliación proviene del latín Conciliatio que significa acción y efecto de conciliar o acuerdo entre los litigantes para evitar un pleito o desistir del ya iniciado (RAE, 2015). Respecto del concepto teoría, habría que decir, como lo define el Diccionario de la Real Academia Española, que "es el conocimiento especulativo considerado con independencia de toda aplicación" (RAE, 2015). Demicheli', hablando de la mediación como forma de comunicación, dice que toda teoría se funda en una epistemología, y que toda teoría, a su vez, media entre la epistemología y la aplicación de una determinada técnica, fundamentos que para el caso que se trata en el presente texto, aplican plenamente, en el entendimiento que se tiene de la conciliación como un mecanismo alternativo de tratamiento de conflictos.

Al efecto, del conocimiento lato que se tiene de la conciliación, quienes ejercen el rol de conciliador, generalmente suelen decir que en su ejercicio siempre aplican una determinada técnica, la misma que ha sido aprendida desde su experiencia o puesta en práctica de dicho mecanismo. Pero, es poco lo que pueden argumentar

\footnotetext{
La palabra epistemología, en apariencia complicada y distante como término, es simple y cotidiana en sus efectos: alude al modo en que percibimos el mundo y nos explicamos las cosas que ocurren en él. Toda teoría se funda en una epistemología, aunque no lo sepamos. Del mismo modo, ninguno de nosotros va por el mundo sin una epistemología acerca de este, aunque no nos percatemos. Toda técnica, deriva a la vez, de una o más teorías, aunque no las conozcamos. Cuando usamos una determinada técnica, entonces, validamos la teoría, aunque no seamos conscientes de ello. Lo abstracto de una epistemología y lo concreto de una técnica está mediado por las teorías; y estas tres instancias en conjunto se entrelazan en nuestro operar diario de una manera mucho más tangible de lo que pareciera a primera vista (Demicheli, 2000, 139).
} 
sobre la teoría que soporta dicha práctica y, menos, cuál es su epistemología.

Así pues, bien podría decirse que para hablar de una teoría de la conciliación, se debe considerar como mínimo: la importancia del entendimiento directo entre las personas, el reconocimiento del otro, la responsabilización del conflicto por cada una de las partes y la posibilidad de su resolución o de su transformación con la presencia y apoyo de un tercero que facilita el proceso comunicacional.

Ahora bien, y en la medida en que se le ha entendido como uno de los principios rectores de la conciliación, y siguiendo el hilo de lo dicho en el párrafo anterior respecto de los fundamentos teóricos de la conciliación, necesariamente se debe hablar de la autonomía de la voluntad. Esta, la autonomía de la voluntad, es uno de los fundamentos que permiten que personas vinculadas por un conflicto puedan decidir sentarse a la mesa de negociación y, sin ninguna afectación de fuerza extraña o coerción, discutan y acuerden sobre lo que les convoca. A este efecto, bien puede citarse lo que el Consejo de Estado en sentencia de 2014, expresó:

[A] pesar de que la autonomía de la voluntad privada suele materializarse en el derecho civil y, específicamente, en el contractual, lo cierto es que este principio rige en todas las actividades humanas donde estén en juego la transacción de bienes o derechos, ya sea de contenido económico o no, por lo tanto, los mecanismos alternativos de solución de conflictos, a excepción del arbitraje, tienen como base para su desarrollo el ejercicio de la autonomía de la voluntad. Tanto los mecanismos alternativos de solución de conflictos autocompositivos como heterocompositivos -exceptuando, como ya se dijo, el arbitrajeconsisten en la manifestación y acuerdo de voluntades para solucionar los mismos y así, sustituir la necesidad de acudir a la jurisdicción. Por lo tanto, la voluntad privada, tiene fuerza normativa, pues a través de ella, las partes del conflicto alcanzan una solución al mismo, mediante una transacción que tiene efectos de cosa juzgada. El caso específico de la conciliación como mecanismo alternativo al judicial, que es el objeto de estudio del presente proveído, consiste en la manifestación de voluntades en la cual interviene un tercero, que si bien, propone fórmulas de arreglo, no tiene la capacidad de imponer una decisión, sino que corresponde a las partes ponerse de acuerdo y consentir o no en la solución propuesta. Entonces, en aplicación del alcance de la autonomía de la voluntad, y la naturaleza de la conciliación, se tiene que la primera manifestación consiste en conciliar o no -ánimo conciliatorio-, posteriormente en definir el contenido del acuerdo, haciendo uso de las capacidades y técnicas de negociación y, por último, aceptar o no la resolución del litigio a la que se llegó.

En conclusión, la naturaleza misma de la conciliación exige el ejercicio pleno de la autonomía de la voluntad y si se logra llegar a un acuerdo, éste tendrá la misma fuerza que una decisión judicial, lo cual vislumbra, una vez 
más, la fuerza jurídica que tiene la voluntad exenta de vicios para producir efectos jurídicos, siempre que respete el orden público y las buenas costumbres, en los términos señalados por la Constitución Política. Ahora bien, la capacidad que tiene la autonomía de la voluntad para producir efectos jurídicos bajo las condiciones y alcances que los particulares definan es perfectamente posible en un contexto privado o comercial, en el que están en juego únicamente intereses particulares de carácter económico o personal, y que no tienen incidencia directa en el devenir de la sociedad (Colombia, Consejo de Estado, 2014).

Ahora, y en la idea de ubicar el enfoque en que debe gravitar la conciliación, en procura de un fundamento firme sobre el cual se asiente el entendimiento de esta como una real posibilidad de alcanzar ciertos niveles de convivencia pacífica, o de una determinada cultura de paz, más allá de su primigenia concepción como mecanismo de descongestión judicial, consideramos que debería ser el "transformativo". Esto, por cuanto el tan socorrido enfoque de "resolución" solo apunta al tratamiento del asunto (Bush y Folger, 1994) o a la simple terminación de un conflicto (Lederach, 1998: 221), mientras que desde el enfoque trasformativo, resaltamos, el tratamiento del conflicto señala el valor de alcanzar una forma genuinamente positiva de conducta humana: la fuerza compasiva, mediante el necesario ejercicio del esfuerzo moral de parte de un individuo para pasar de un estado de debilidad o egoísmo, a uno de fuerza y compasión.
La transformación es, pues, valiosa, tanto a causa de la elevada bondad de la conducta humana que es su resultado, como del gran esfuerzo moral necesario para obtenerla (Bush y Folger, 1994: 339). Para París (2005: 158), por su lado, la denominación de transformación de conflictos es la que mejor se relaciona con los presupuestos de los estudios para la cultura de paz. Es la mejor forma para regular un conflicto. Este enfoque permite hacer una regulación positiva del mismo, convirtiendo las situaciones conflictivas en situaciones de aprendizaje en las que priman la comunicación, el reconocimiento del otro, el respeto, la responsabilización del conflicto, el diálogo y el afecto.

Roldán (2007), por ejemplo, expresa que los conceptos de solución y resolución deben asumirse diferentes. El primero hace alusión a la solución de problemas; sin embargo, este pierde la perspectiva del conflicto y no va más allá del hecho de que el conflicto no es solo una situación problemática. El segundo, en cambio, tiende a regular la relación social y pone en evidencia que resolver un conflicto no es solo tratar un problema, sino profundizar e intervenir desde la raíz el conflicto, regulando, de contera, las relaciones sociales.

En el tratamiento o resolución de conflictos, se debe tener en cuenta la teoría de reconocimiento del otro, que permite asumir al otro en una entidad tal que su reconocimiento es igual al yo (Roldán, 2007). Respecto de esta teoría, como bien lo apuntan Bush y Folger (1994), en su fundamentación del enfoque de mediación 
transformativo, no se puede dejar de lado precisamente la otra cara de la moneda, la revalorización o la valía propia de cada una de las personas vinculadas con el conflicto. Así, pues, en la conciliación con miras a la transformación, la respuesta ideal a un conflicto no consiste en "resolver el problema", sino en ayudar a transformar a los individuos comprometidos, en las dos dimensiones del crecimiento moral: el fortalecimiento del yo, y la superación de los límites del yo para relacionarse con otros.

De forma similar, Ripol-Millet (2001) anota que el tratamiento del conflicto, desde la perspectiva transformativa, es una oportunidad para lograr dos objetivos fundamentales para la madurez y el crecimiento personal:

1) El reconocimiento del otro, que fortalece la propia capacidad como individuo para experimentar y expresar consideración y preocupación por los demás, especialmente cuando su situación es diferente a la propia y, 2) El fortalecimiento propio, que aumenta la capacidad como individuo para afrontar las circunstancias adversas y los problemas de todo tipo (RipolMillet, 2001: 47-48).

Para Vinyamata (2000), los conflictos forman parte de una manera de hacer, de una historia y de una percepción y actitud concretas. Contribuir a modificar la percepción personal del entorno que se habita puede significar empezar a cambiar el curso de los acontecimientos. Es así como transformar las percepciones, ac- titudes y comportamientos puede influir poderosamente en la transformación de situaciones conflictivas cuyas soluciones viables no se acierta a ver.

Así, entonces, la resolución de conflictos no se plantea como una utopía, por cuanto esta no se piensa de manera estructural, como en la idea marxista del fenómeno, en donde el conflicto social podría resolverse en la medida en que se superara la división de las clases sociales. Por tanto, el tratamiento de los conflictos no debe tender en su proyección ineludiblemente hacia adelante, los conflictos no simplemente se resuelven o solucionan, sino que también se transforman; esto, desde la propuesta o enfoque transformativo presentada por Bush y Folger (1994), ya que el proceso desde esta perspectiva es intemporal, pues en el desarrollo del proceso, se pueden representar aparentes retrocesos, pero siempre, desde la denominada técnica de los microenfoques aplicada al valor que contiene cada una de las contribuciones ofrecidas por las partes, ha de entenderse siempre, que dichos aportes o contribuciones son como agentes que impulsan el movimiento progresivo del proceso transformador (Bush y Folger, 1994: 296).

\section{LA EXPERIENCIA DE LOS ACTORES EN EL PROCESO CONCILIATORIO}

A efectos de abordar las evidencias que arrojó la investigación respecto de la experiencia de los actores en el proceso conciliatorio, bien vale la pena referir, a modo descriptivo, los conflictos de que conoce dicho centro de conciliación y 
la forma más socorrida por estos para abordarlos. En términos generales, los conflictos conocidos por el Centro de Conciliación de la Universidad de Antioquia no se apartan de la exigencia de ley que los regula, es decir, este centro conoce, en principio, de los denominados conflictos de índole civil y de familia que, a su vez, gozan de las cualidades de ser disponibles o transigibles, según el mismo interés de las partes.

De los asuntos conflictivos en materia de familia que conoce regularmente este Centro de Conciliación, según lo referido por Montoya, Salinas, Osorio \& Martínez (2011), se pueden citar: 1) fijación de cuota alimentaria para menores o incapaces, o mayores en estado de necesidad; 2) custodia y visitas de menores e incapaces; 3 ) salidas de menores del país; 4) separación de bienes y de cuerpos; 5) establecimiento de la existencia de la unión marital de hecho y de la sociedad patrimonial entre compañeros permanentes; 6) disolución y liquidación de la sociedad conyugal; 7) disolución y liquidación de sociedad patrimonial entre compañeros permanentes; 8) bienes objeto de sucesión y administración de estos entre herederos, y 9) dirección conjunta del hogar entre cónyuges y compañeros, y entre padres sobre el ejercicio de la autoridad paterna o la patria potestad.

De los asuntos en materia civil, por su lado, se pueden citar como ejemplo, los siguientes conflictos: 1) entre arrendador y arrendatario respecto del incumplimiento del contrato; 2) entre poseedores o propietarios sobre los linderos de sus predios; 3) reclamación de perjuicios por responsabilidad civil contractual o extracontractual; 4) por el incumplimiento de contrato de compraventa de bienes muebles o inmuebles; 5) sobre servidumbres; 6) por el incumplimiento en el pago de créditos o del contrato de mutuo; 7) entre propietarios o poseedores de bienes inmuebles colindantes respecto del uso de muro medianero; 8) entre propietarios o poseedores de bienes inmuebles colindantes respecto de humedades o peligro de derrumbamiento; 9) por mejoras en bien ajeno; 10) entre copropietarios por la violación de los derechos en los espacios de uso común y 11) sobre liquidaciones de sociedades de hecho.

En cuanto a las formas en que las partes solían atender sus asuntos conflictivos antes de acceder a la conciliación (Montoya, Salinas, Osorio \& Martínez, 2011), las respuestas más recurrentes se sintetizan en:

En casi todas las respuestas dadas, las partes expresan que la mejor forma de afrontar o darles solución a los conflictos es el diálogo, pero la realidad muestra lo contrario, pues generalmente asumen una actitud que niega lo dicho y que evidencia el desconocimiento o la ignorancia que tienen sobre la comunicación. Es decir, más que hablar y escuchar, lo que hacen es denostar y descalificar al otro.

Respecto del tratamiento del conflicto, antes de acudir al Centro, las partes consultadas se manifiestan en la siguiente perspectiva: cuando tienen que asumir 
la resolución de un conflicto, no dudan en manifestar que lo hacen desde la búsqueda del diálogo. Se evidencia entonces la necesidad de expresar los sentimientos y de ser escuchados, comprendidos y reconocidos por el otro, cosa que frecuentemente no se consigue satisfactoriamente, por cuanto se desconocen o se violentan los canales propios de la comunicación (p. 124).

Así, pues, de los datos recopilados y analizados en la investigación (Montoya, Salinas, Osorio \& Martínez, 2011), la voz de los actores involucrados cobró inusitada importancia y se dejó escuchar con suficiente propiedad, respecto de la manera en que asumen la conciliación y el proceso mismo de conciliación. Las partes, luego de terminado dicho proceso, en términos generales expresaron las nuevas formas de percibir al otro y el conflicto, así como las nuevas posibilidades de confrontar o conversar con la contraparte en la idea de un acercamiento más franco y transparente en pro de la solución o transformación del conflicto y su relación con ese otro. Los conciliadores, por su lado, propusieron un ámbito particular de relacionamiento en el proceso conciliatorio, de acuerdo con el conocimiento previo adquirido en su formación profesional y en especial en la denominada cátedra Teoría de la conciliación impartida en el Programa de Derecho.

Hablar de la transformación de los conflictos en la conciliación podría parecer extraño al objeto del mecanismo. Pero, con base en lo observado en los procesos de conciliación analizados y en los resultados evidenciados en los intervinientes, nada más alejado de ese prejuicio. La transformación del conflicto en la conciliación deriva básicamente de la formación y de la preparación del conciliador, y del tratamiento que del conflicto y la relación de las partes este implemente en el proceso de conciliación. Con el mero hecho de que las partes acepten el encuentro, a sabiendas de sus posturas e intereses, obviamente opuestos, ya se inicia la apertura de una posible actitud de cambio en las relaciones y en el entendimiento del conflicto. Si bien el proceso de conciliación en el Centro de Conciliación de la Universidad de Antioquia, se inicia y se termina ajustado a las exigencias de ley, en este se ha venido evidenciando un elemento que a todas luces es de relievar por su importancia: la técnica. En este proceso y en específico en la audiencia de conciliación, el conciliador ha venido implementando una técnica de cierta conformación ecléctica (Giraldo Montoya, 2004, citada por Montoya, Salinas, Osorio E Martínez, 2011), en la que se funden la teoría de la "Escuela de Negociación de Harvard" y la teoría del Modelo Transformativo de Bush y Folger (Demicheli, 2000).

Así pues, al tiempo que el conciliador propicia el encuentro de las partes a efectos de que ellos directamente aborden la solución del conflicto que los enfrenta ${ }^{2}$, de igual forma, dispone de las herramientas

\footnotetext{
El énfasis para superar el conflicto en este enfoque, está puesto en el contenido de lo que se está tratando y no en la relación entre las personas. El modelo señala la necesidad de separar las personas del problema.
} 
del enfoque transformativo ${ }^{3}$, en la idea de que el proceso conciliatorio no pase de ser el simple cumplimiento de un requisito para acceder a la vía judicial. En el desarrollo de la audiencia, entonces, los conciliadores, en el ejercicio de su rol, alcanzan a aplicar uno y otro modelo, dando, al final, una amplitud y una flexibilidad tales al proceso, que permiten que ambas posibilidades se asomen a las opciones de tratamiento del conflicto: la resolución o la transformación o, aun, que se llegue a resolver el conflicto (en el campo eminentemente del asunto) y que la relación de las partes, en mayor o menor medida, se transforme o modifique.

Según lo observado en la investigación de referencia, otra de las herramientas utilizadas por el conciliador para el desarrollo de la audiencia de conciliación es el denominado proceso pedagógico, el cual se inicia desde las acciones previas a la audiencia por parte del conciliador. Este proceso comprende no solo el hecho de informar a las partes qué es la conciliación y cuáles son sus ventajas, límites y efectos, sino también el de generar en las partes, mucho antes de que se sienten a la mesa de conciliación, la conciencia de que el conflicto es de ellos, que en ellos

3 El objetivo de este modelo es transformar la relación entre las partes, más allá de la consecución de un acuerdo o la resolución del conflicto. En este modelo las herramientas o elementos de que se disponen, son la revalorización y el reconocimiento. Entendida la primera como la oportunidad que se dan las partes de identificarse en su propia valía y de encontrarse precisamente con el otro, desde el reconocimiento. Ver: Guido Demicheli M. "Comunicación y Modelos de Mediación: epistemología, teoría y técnicas". En Revista Estudios sociales N. ${ }^{\circ}$ 106, págs. 141 y 144. mismos se encuentra la solución y que se debe reconocer al otro en la misma dimensión en que se tiene la valía propia, como persona y como interviniente.

En la perspectiva de abonar más elementos pedagógicos al proceso de conciliación, a las partes se les instruye antes y durante la audiencia de conciliación, sobre el hecho de que en la confrontación o en la conversación [como prefieren llamarla Suares (2002) y Muldoon (1998)], entendida como la importancia del enfrentamiento o del encuentro cara a cara con quien se asume como el adversario, la solución de los conflictos siempre se podrá abordar, ora directamente (con la ayuda de un tercero facilitador), ora por medio del fallo de un tercero autorizado para ello (el juez). En ambos campos, según lo expresa el mismo Muldoon (1998), a la primera forma le denomina vía alta y a la segunda vía baja. La vía alta es la posibilidad del entendimiento directo de las personas, basadas en que se reconoce la fuerza del adversario, la confianza que se puede depositar en él y que ambas partes aceptan el desafío de la confrontación y el potencial de la transformación del conflicto. En la denominada vía baja, queda la alternativa de acudir al juez, en el entendido de que en el adversario no se puede confiar y que este se niega a hacerse responsable de sus actos o no acata la ley o los acuerdos.

Por otro lado, es decir, desde la perspectiva de las partes, estas, según lo derivado de la investigación, propusieron el entendimiento del fenómeno de 
transformación, de acuerdo con su participación en el proceso de conciliación: la parte citante, por el hecho de haber participado de un proceso pedagógico ${ }^{4}$ previo a la audiencia de conciliación, en la cual se brindó la posibilidad, para el momento de confrontación con el otro, reconoció el campo de acción en el que se desenvolvería para poder lograr su propósito conciliatorio; la parte citada, en cambio, en su condición de invitada, expectante, evidenció la asunción de dicha experiencia de forma diferente. Esto, por cuanto para dicha parte no se logró, en términos generales, hacerle más propicio el acceso previamente al proceso pedagógico, como sí lo tuvo el citante; aquí, se observó que el citado intentó actuar de acuerdo con la situación que le propusieron en el escenario de la conciliación, más, cuando el conciliador en la audiencia planteó el sentido y el propósito de la conciliación, las reglas que se deben seguir en la audiencia de conciliación y su dinámica. Con ello, según los resultados finales de la investigación, se logró poner de presente en el espacio mismo de la conciliación y en el entendimiento de todos sus intervinientes, que dicha dinámica conciliador-partes-proceso genera un campo de actuación tan especial o específico, en el cual todos los actores pudieron evidenciar que allí siempre se

4 El proceso pedagógico es un elemento fundamental en el proceso de conciliación, este se da antes y durante la audiencia de conciliación y consiste en la instrucción brindada por el Centro de Conciliación y el conciliador a las partes en conflicto, respecto de lo que es este mecanismo, sus características, alcances y la importancia que como personas tienen en este proceso las partes. podrán encontrar, desenvolver y reconocer.

Con lo dicho, entonces, bien podría decirse que, al igual que las relaciones sociales, con sus características intersubjetivas, la conciliación es también un proceso dinámico, un progresivo desarrollo dialéctico de personas vinculadas por un conflicto que puede o no finalizar con un acuerdo, pero que, en sí, siempre conservará el potencial de la transformación de las personas, de su relación y de la concepción que tengan del conflicto mismo. Mediante la interacción de los sujetos, se entrelazan formas particulares de ver el mundo, y, por tanto, el contacto cara a cara es solo un elemento del proceso, pero, tal vez, uno de los más importantes.

El proceso de conciliación se realiza necesariamente mediante la interacción que facilita el conciliador, en la relación que establece con los conciliantes para posibilitar su encuentro en la arena o escenario de la conciliación, donde no solo prima la palabra sino también la construcción simbólica del lenguaje no verbal (Montoya E Salinas, 2008). En cualquier relación específica que se prolongue, habrá momentos de acuerdo y momentos de confrontación, en donde cada parte debe, en alguna medida, mostrar deferencia para con la otra, a efectos de que la interacción sea posible.

La conciliación se da porque ambas partes reconocen, con el apoyo del facilitador, que tienen intereses comunes, si no similares, que les posibilitan un acercamiento. En este sentido, las partes se re- 
conocen en una relación interdependiente en mayor o menor medida la una de la otra para conseguir sus metas (Beltri, 2000). En contraste con la fuerza puramente negativa de la indiferencia (Greatbach \& Dingwall, citados por Folger $\&$ Jones, 1997: 127), el conflicto proporciona un incentivo para la interacción, consista ella en unirse o en oponerse a los intereses del otro.

En cuanto al rol de conciliador, asumido como elemento estructural del proceso, a efectos de los propósitos o fines perseguidos por este, es de vital importancia lo que este conciba como conciliación, pues de esto depende en gran medida la forma como asume su tarea, la manera como se responsabiliza de su actuar (Vásquez, 2010) y del apoyo que brinda a las partes para la resolución o transformación de los conflictos (Montoya, Salinas, Osorio E Martínez, 2011).

Según lo observado en la investigación, a los conciliadores del referido Centro no les es extraño asumir su responsabilidad y el entendimiento de la figura de la conciliación de forma diferente a la que tradicionalmente se le ha querido asignar como simple mecanismo de descongestión judicial (Colombia, Ministerio de Justicia y del Derecho, Dirección de Conciliación, 2016) $)^{5}$. Alguno de los conciliadores, por ejemplo, expresó: "La finalidad de la conciliación es servir de

5 Con la expedición de la Ley 23 de 1991, el Legislador quiso en el espíritu de dicha norma, crear un mecanismo de descongestión de despachos judiciales. No se pensó sino en atender la crisis de la administración de justicia por el alto índice de trabajo que afrontaba la rama judicial a causa de la congestión. método alternativo de resolución de conflictos en el cual cada parte cede un poco en sus intereses para llegar a un acuerdo satisfactorio" (Entrevista a estudiante conciliador, tomado de Montoya, Salinas, Osorio \& Martínez, 2011).

Para la mayoría de los conciliadores consultados, el ejercicio de sus funciones se reduce al papel desempeñado en el desarrollo de la audiencia. Sin embargo, para otros, dicho rol no se limita a tal momento, sino que se debe entender desde la recepción del asunto y la atención de los usuarios. En estos casos, podría decirse, el conciliador percibe su rol y la conciliación misma, como un proceso integral, en el que se debe participar de principio a fin.

Algunos conciliadores del Centro objeto de la investigación, según lo analizado, expresaron que conciben la conciliación como un proceso que, además de propiciar la resolución de los conflictos, contribuye a la formación de las partes sobre una concepción más holística del conflicto, sobre la responsabilización del mismo y de las formas de resolverlo, distintas a las vías de hecho o a la vía judicial:

Mi función es orientar a las partes en conflicto, a un posible acuerdo buscando mecanismos de educar a las partes en que los problemas se pueden arreglar dialogando y sin acudir a otras instancias judiciales (Entrevista a estudiante conciliador, tomado de Montoya, Salinas, Osorio E Martínez, 2011). 
En este orden de ideas, el conciliador ha de seguir un procedimiento que favorezca la revisión de las contribuciones de cada parte al surgimiento del conflicto y también resaltar el modo en que los disputantes aportan al mantenimiento del conflicto durante la conciliación misma. Es por esto que se hace pertinente la identificación del estado de las relaciones como tal y su afectación por el conflicto.

En este sentido, huelga decir, existen variaciones en las relaciones entre las partes antes de la audiencia y después de ella. Esto es, se puede dar que las partes entren a la audiencia sin hablarse, escépticas, enojadas, con prevenciones y salir, al final, con una actitud totalmente contraria. También puede suceder que las partes entren disgustadas y salgan de la audiencia de la misma forma. O puede ocurrir que al inicio tengan una actitud conciliadora, respetuosa y cordial, y salgan de la misma, sin modificación alguna al respecto. Esto se puede presentar independientemente del resultado del proceso conciliatorio; es decir, si hay o no un acuerdo, o si el acuerdo es parcial. Pero lo que sí es claro es que en ello el conciliador juega un papel importante como director de la audiencia y como quien debe establecer una comunicación fluida y clara entre las partes (Ortuño y Hernández, 2007:13), ya que él se encuentra, ante todo, legitimado para ejercer su rol mediador y abrir las puertas a un posible cambio o transformación del conflicto o de la relación de las personas.

Respecto a las partes, la relación que establecen entre sí tiene gran relevancia cuando se desea desentrañar el origen de una situación conflictiva, por cuanto este aspecto influye en el desarrollo y resultado del proceso conciliatorio. De ahí, el hecho de que el replanteamiento de dicha relación y del conflicto mismo sea indispensable para su transformación y para la validez de la aplicación del enfoque transformativo, dado que resulta preponderante y significativo el aspecto relacional en el tratamiento de los conflictos, en oposición, por supuesto, al enfoque resolutivo, pues este se circunscribe, como ya se había dicho, solo al asunto y no tanto a las personas.

Se evidencia en los hallazgos de la investigación de que se trata que, en el proceso conciliatorio, se aprecian actitudes y comportamientos de las partes que se transforman durante el desarrollo de las audiencias de conciliación. En un primer momento, al inicio de la audiencia, generalmente las partes llegan con diferentes actitudes, dependiendo básicamente de su condición de citante o de citado; las actitudes más comunes suelen ser de sorpresa, descontento, expectativa, agresividad, ofensiva; muy escasamente se encuentran actitudes calmas o positivas o de "conciliación". En un segundo momento, cuando las partes manifiestan su versión de los hechos, afloran los sentimientos y la forma como cada quien ve el mundo y al otro, mediante manifestaciones de llanto, silencios, agresividad o insultos.

El tercer momento de dicho proceso comienza cuando la etapa anterior se agota plenamente y el conciliador facilita 
que las partes reconozcan sus intereses y propongan salidas negociadas al conflicto. Una vez terminado esto, y según lo hallado en la investigación, la actitud de los actores frente al conflicto y frente al otro, en la mayoría de las veces cambia, en el sentido de que ya se aprecian capaces de comunicarse y tienen una percepción más amplia de su conflicto y del otro, pues se dieron la oportunidad de verlo más como una oportunidad de mejorar que como un problema.

De ahí que las partes se conviertan en multiplicadoras de las posibilidades que ofrece la conciliación para la transformación de los conflictos y de una nueva cultura de paz o de convivencia pacífica. El hecho de que se pueda apreciar directamente por los actores involucrados en un conflicto que se pueden encontrar, conversar, reconocerse, discutir ampliamente sobre los intereses que los enfrentan y de que puedan sondear cualquier cantidad de posibles soluciones, acompañados de un tercero facilitador, es fiel muestra de la posibilidad de potenciar su capacidad de autogestionarse y de reflexionar sobre otras formas de tratar los conflictos, en el mismo sentido que anota Fisas (2004)

[...] la paz es algo más que la ausencia de guerra, y tiene que ver con la superación, reducción o evitación de todo tipo de violencias, y con nuestra capacidad y habilidad para transformar los conflictos, para que, en vez de tener una expresión violenta y destructiva, las situaciones de conflicto puedan ser oportunidades creativas, de encuentro, comunicación, cambio, adaptación e intercambio. Este nuevo enfoque es el que persigue la "cultura de paz", o "cultura para la paz", si la entendemos como un proceso que, en primera instancia, habrá de transformar la actual "cultura de la violencia" (p. 349).

\section{CONCLUSIONES O HALLAZGOS}

El acercamiento al conflicto y su tratamiento desde una perspectiva diferente a la simple descongestión de despachos judiciales hace ver, definitivamente, en la conciliación un mecanismo con un potencial tal de transformación que indudablemente se muestra como motor de cambio en la conciencia de los individuos respecto de la gestión de sus propios conflictos y de la importancia del otro como interlocutor válido.

La referida investigación arrojó, como hallazgo especial, que a medida que el proceso de conciliación se desarrolla los imaginarios de quienes están vinculados por un determinado conflicto van cambiando, pues cada uno de los pasos establecidos o acordados en dicho proceso, para llegar al restablecimiento de las relaciones fracturadas por el conflicto mismo, hacen parte de otro proceso tácito, pero permanente, el pedagógico, el cual permite, según las interacciones que allí se presentan, ampliar la capacidad de pensamiento de acuerdo con las dinámicas que se propician en la relación de las partes al momento de tratar directamente sus conflictos.

En el intercambio de ideas generado en el proceso conciliatorio, las personas apren- 
den "significados y símbolos para ejercer su capacidad de pensamiento y de actuar e interactuar" (Ritzer, 2002), permitiendo al conciliador la posibilidad de transformar o afectar las significaciones y los símbolos que las personas emplean en la acción y la relación intersubjetiva sobre la base de la interpretación que hacen del conflicto que les convoca, en específico, en la audiencia conciliatoria. "Las personas son capaces de introducir estas modificaciones y alteraciones debido a su capacidad para interactuar consigo mismas, lo que les permite examinar los posibles cursos de acción y valorar sus ventajas y desventajas relativas para luego elegir uno" (Ritzer, 2002: 271) que facilite el acercamiento a una solución viable al conflicto.

Los imaginarios frente al conflicto y la conciliación varían o se transforman en la conciliación en la medida en que también se construye ciudadanía a través de la participación en este proceso de resolución negociada de conflictos, por medio de la relación con el otro, en la toma de conciencia de la importancia de tener en cuenta y entender el entorno, los intereses y las expectativas que las personas tienen en determinado conflicto. Se construye ciudadanía en la medida en que las partes acceden a información y forman su propio criterio respecto a ella, porque la conciliación genera un espacio incluyente donde se argumenta, se escucha, se debate, se negocia, se discute, se reconoce al otro y se acuerda, elementos presentes en todo proceso participativo o de interacción.

Con todo lo anotado, se concluye, entonces, que la dimensión de la transforma- ción en el tratamiento de los conflictos mediante el proceso de conciliación trasciende a la mera estipulación legal, al espíritu mismo que el legislador le predestinó en su creación (mecanismo descongestionador de despachos judiciales) y trasciende igualmente el entendido de que con la resolución se terminan o eliminan los conflictos. Así, pues, cuando se habla de resolución de conflictos, se hace un especial énfasis en mecanismos alternativos; sin embargo, en la dimensión que se pretendió evidenciar, pensar que los conflictos o las relaciones de las personas vinculadas con el conflicto se transforman es reconocer al otro y su representación o imaginario sobre el conflicto, suponiendo que la visión y percepción de los actores debe ser vista siempre de otra forma: la del cambio, la transformativa.

\section{REFERENCIAS BIBLIOGRÁFICAS}

BELTRI, F., (2000). Aprender a negociar, Barcelona: Ediciones Paidós Ibérica.

BUSH, B. E FOLGER, J.P., (1994). La promesa de mediación. Barcelona: Granica.

COLOMBIA, Consejo de Estado, 2014, Sentencia No 07001-23-31-000-2008-00090-01(37747) - Sección Tercera, de 24 de noviembre de 2014 [en línea], disponible en http://consejoestado.vlex.com.co/vid/569540426, consulta octubre 20 de 2016.

COLOMBIA, Corte Constitucional, 2001, Sentencia C-1195, Demanda de inconstitucionalidad contra los artículos 35, 36, 37, 39 y 40 de la Ley 640 de 2001 "Por la cual se modifican normas relativas a la conciliación y se dictan otras disposiciones", Bogotá, 15 de noviembre, Centro de Arbitraje y Conciliación Cámara de Comercio de Bogotá [en línea], dis- 
ponible en http://www.cacccb.org.co/documentos/94 sentencia 1195.pdf, consulta mayo 12 de 2005.

Colombia, Ministerio de Justicia y del Derecho, Dirección de Conciliación, [en línea], disponible en: http://conciliacion.gov.co/portal/conciliacion/conciliacion-definicion, consultada octubre 20 de 2016.

DEMICHELI, G., (2000). Comunicación y modelos de mediación: Epistemología, teoría y técnicas, en Revista de Estudios Sociales, 106/ Trimestre 4/ 2000, Santiago de Chile: Corporación de Promoción Universitaria.

FISAS, V., (2004). Cultura de paz y gestión de conflictos. Ediciones UNESCO. Cuarta edición. España.

FOLGER, J., \& JONES, T., (1997). Nuevas direcciones en mediación. Barcelona. Paidós.

GARCÍA, B., et al, (2002). Técnicas interactivas para la investigación social, Medellín: Fundación Universitaria Luis Amigó.

LEDERACH, J. P., (1998). Un marco englobador en la transformación de los conflictos sociales crónicos, Ed. Guernica Gogoratuz, Documento N. ${ }^{\circ} 12$, Biskaia, España.

MONTOYA, M. Á. E SALINAS, N. A., (2008). La guerra de los valientes o el lugar de la conciliación. El escenario de los actores de la conciliación: construcción del lugar simbólico, en Revista Estudios de Derecho, Medellín: Universidad de Antioquia.

MONTOYA, M. Á; SALINAS, N. A.; OSORIO, B. F.; E MARTÍNEZ, S. M., (2011). Teoría y práctica de la conciliación, Medellín: Editorial Universidad de Antioquia.

MULDOON, B., (1998). El corazón del conflicto. 1. ${ }^{a}$ edición Buenos Aires: Editorial Paidós.

ORTUÑO, J. E HERNÁNDEZ, J., (2007). Sistemas alternativos a la resolución de conflictos (ADR): la mediación en las jurisdicciones civil y penal.
Documento de trabajo 110/2007. (http:// www.falternativas.org/index.php/content/ view/440/68/; página consultada el 3 de abril de 2008).

PARÍS, S., (2005). La transformación de los conflictos desde la filosofía para la paz. (http://www.tdx. cat/bitstream/handle/10803/10456/paris. pdf?sequence $=1$, página consultada el 18 de septiembre de 2008).

REAL ACADEMIA ESPAÑOLA. Diccionario de La Lengua Española (http://www.rae.es/ rae.html, página consultada el 18 de julio de 2016).

REPÚBLICA DE COLOMBIA, Presidencia de la República, Ministerio de Justicia y del Derecho, 1997, Proyecto de Ley N. ${ }^{\circ} 234$ de 1996 (Cámara). Justicia alternativa para lograr la paz. Bogotá, marzo.

RIPOL-MILLET, A., (2001). Familias, trabajo social y mediación, Barcelona: Paidós.

RITZER, G., (2002). Teoría sociológica moderna, $5^{\mathrm{a}}$ ed., Madrid: Mc. Graw Hill.

ROLDÁN, H., (febrero 26 al 28 de 2007). Teorías sobre las formas de tratamiento de conflictos, en ENCUENTRO NACIONAL. Las rutas académicas de los MARC en Colombia, Medellín: Universidad de Antioquia.

SCHAEFER, R., (2006). Introducción a la sociología, Madrid: Mc Graw Hill.

SUÁREZ, M., (2002). Mediación. Conducción de disputas, comunicación y técnicas. Buenos Aires: Paidós.

VÁSQUEZ, R., (2010). La resolución de conflictos familiares, en Revista Justicia Juris, ISSN 1692-8571, Vol. 6. N. ${ }^{\circ}$ 13. Abril-diciembre, Pp. 40-48.

VINYAMATA, E., (2000). Aprender mediación. Barcelona: Paidós Ibérica. 